\title{
Développement des plants de hêtre (Fagus sylvatica $L$ ) dans une régénération naturelle, équienne, âgée de 18 ans
}

\author{
B Thiébaut 1, 2, *, B Comps 3, M Rucart 1, \\ S Soroste 1, C Ntsame Okwo 1
}

\author{
1 Université des sciences et techniques du Languedoc, institut de botanique, \\ 163 rue Auguste-Broussonnet, 34000 Montpellier; \\ 2 CNRS, centre Louis Emberger, BP 5051, 34033 Montpellier Cédex; \\ ${ }^{3}$ Université de Bordeaux I, département de biologie des végétaux ligneux, \\ avenue des facultés, 33405 Talence, France
}

(Reçu le 15 juillet 1991; accepté le 19 novembre 1991)

\begin{abstract}
Résumé - Dans une régénération naturelle de hêtre, équienne, âgée de 18 ans, le développement des plants est étudié en analysant la morphologie de leur tige principale, sous 3 éclairements : à la lumière, en demi-lumière et à l'ombre. La régénération est pluristrate dans les 2 premiers cas et monostrate dans le dernier. La structure se dessine tôt et son caractère mono- ou pluristrate apparaît rapidement. La stratification a une influence sur le développement des plants à l'âge de 18 ans ainsi que sur leur croissance annuelle et cumulée, chaque année pendant 18 ans. Les reclassements des individus seront examinés dans un prochain article.
\end{abstract}

régénération naturelle $/$ Fagus sylvatica $=$ hêtre $/$ stratification $/$ croissance

Summary - Growth of young beeches (Fagus sylvatica $\mathrm{L}$ ) in an even-aged, 18-year old natural beech regeneration. In an even-aged 18-year old natural beech regeneration, the growth of young plants has been analysed using only the main stem under 3 daylight intensities: full-light, halflight, and shade. The structure shows 3, 2 and 1 layers respectively under these light conditions (table I). The variations in total height and root collar diameter had 2 components in full-light and in half-light only, ie among-and within-layers (figs 1, 2 and 3). In full-light, the height variations were responsible for the differences among the layers and those of root collar diameter for the differences within the layers, fig 1. In half-light the variations in height and root collar diameter were simultaneously responsible for the differences among and within the layers (fig 2). The structure appeared early with a single or several layer(s) (fig 8). The annual and multiannual growth exhibited 3 periods: establishment, differentiation, and regression or stabilisation, (figs 5, 6, 8 and 9). In full-light where differentiation is best between the layers, stratification had an influence on the vigour of the seedlings and to some degree on their shape, but not in half-light (fig 10). The layers may be considered as reflecting social status in full-light where the height is regulated by stratification. The classification of seedlings according to their total height over the 18-year period will be analysed in a subsequent paper.

natural seedling / Fagus sylvatica = beech / stratification / growth

\footnotetext{
* Correspondance et tirés à part
} 


\section{INTRODUCTION}

La croissance du hêtre est encore peu connue pendant les 20 premières années de son développement en forêt. Pourtant cette période est primordiale dans la vie d'un arbre et pour l'avenir du peuplement. En effet, c'est à ce moment que les individus commencent à se différencier les uns des autres par des développements inégaux (Falcone, 1985; Thiébaut et al, 1985).

La diversité morphologique des jeunes hêtres est influencée par des facteurs d'ordre génétique, écologique, sylvicole et même aléatoire (Krahl-Urban, 1962; Galoux, 1966; Teissier-du-Cros, 1981; Teissier-du-Cros et Thiébaut, 1988; Cuguen et al, 1988; Comps et al, 1988). Parmi les facteurs du milieu, la lumière et la densité de la régénération jouent un rôle important sur le développement des arbres.

La luminosité favorise la croissance mais il existe un optimum qui varie selon le climat régional (Aussenac et Ducrey, 1977; Lanier, 1981a; Thiébaut, 1982; Dupré, 1984; Hubert, 1984; Dupré et al, 1986; Urechiatu, 1986). La densité des jeunes peuplements a des effets positifs : elle accélère la croissance individuelle en hauteur et favorise les disparités de développement (Hubert, 1968; Schütz, 1981; Becker, 1981). Mais elle a aussi des effets négatifs quand elle devient excessive car elle freine le développement des individus, y compris celui des plus grands (Delvaux, 1964 et 1971; Lanier, 1981b; Polge, 1983; Falcone et al, 1986).

Les différences de développement entre individus sont plus importantes à la lumière, elles augmentent avec la densité et avec l'âge de la régénération (Delvaux, 1975; Polge, 1983; Falcone et al, 1986). Ainsi, la lumière et la densité accélèreraient le classement des plants selon leur vigueur et favoriseraient l'apparition d'une structure pluristrate (Delvaux, 1964, 1966 et 1975; Schütz, 1981; Falcone et al, 1986; Lanier, 1986; Zarnovican et Trencia, 1987). Dans les peuplements adultes, ces stratifications paraissent stables. En est-il de même dans les régénérations où le classement d'un jeune arbre semble moins définitif que celui d'un adulte, surtout chez les espèces tolérant l'ombre comme le hêtre ? Nous allons analyser le fonctionnement d'une régénération de hêtre en portant notre attention sur 4 points :

- les différences de développement interet intrastrate,

- l'influence d'une stratification sur le mode de croissance des individus,

- le délai nécessaire pour mettre en place une structure pluristrate,

- et, enfin, la stabilité de cette structure.

Les 3 premiers points seront abordés ici en analysant le développement de plants dans une régénération équienne de 18 ans. Le dernier sera examiné plus tard, en considérant les reclassements des plants selon leur hauteur totale, chaque année pendant 18 ans.

\section{MÉTHODES}

\section{Situation écologique de la régénération}

Au massif de l'Aigoual $\left(44^{\circ} 20^{\prime} \mathrm{N}-3^{\circ} 60^{\prime} \mathrm{E}\right)$, dans la forêt du Lingas située à $1100 \mathrm{~m}$ d'altitude sur un versant exposé au nord ( $20 \%$ de pente), au sol brun forestier acide sur granite, des parcelles ont été mises en régénération après une coupe d'éclaircie en 1968. En 1986 au moment des observations, 2 générations coexistaient : des arbres adultes de 100 à 150 ans, de $15 \mathrm{~m}$ de haut en moyenne, et des jeunes de 18 ans, issus de semis après l'éclaircie. Aucune opération culturale n'a été effectuée dans ce peuplement entre 1968 et 1986.

À cette altitude le climat montagnard est rigoureux et caractérisé par une température 
moyenne annuelle de $4,7^{\circ} \mathrm{C}$ (Garnier, 1967) et des précipitations moyennes annuelles de 2165 $\mathrm{mm}$. Le régime pluviométrique est méditerranéen, avec un déficit estival prononcé (Thiébaut, 1979).

\section{Description du dispositif}

La régénération naturelle a une répartition discontinue. Sa densité peut atteindre jusqu'à 300 individus par $\mathrm{m}^{2}$ et correspond plutôt à celle de semis âgés de 1-3 ans (Suner et Röhrig, 1980; Oswald, 1981). Au même âge, les densités indiquées sont beaucoup plus faibles, comprises entre 50000 et 100 000/ha (Suner et Röhrig, 1980; Falcone et al, 1986).

Un transect d'observation a été installé dans une zone où la régénération est équienne, condition indispensable pour décrire la différenciation morphologique progressive des individus (Van Miegroet et Lust, 1972; Schütz, 1990; Thiébaut et Comps, 1992).

Les semenciers situés au-dessus de la régénération créent des zones d'ombre et de lumière. Le transect traverse 3 zones situées respectivement à l'ombre, en demi-lumière et en pleine lumière. Aux 2 extrémités du transect, de la lumière vers l'ombre, l'extinction de l'éclairement atteint $90 \%$ vers midi, par temps clair, en phénophase feuillée, entre 400 et $700 \mathrm{~nm}$. Les autres conditions du milieu : sol, microrelief et végétation adventice paraîssent homogènes. Tous les individus touchant un ruban métallique tendu sur $55 \mathrm{~mm}$ ont été étudiés. Leur densité a été estimée par le nombre de sujets par $m$ linéaire.

La structure verticale d'une régénération se manifeste physionomiquement par l'apparition d'une strate (structure monostrate) ou de plusieurs strates (structure pluristrate) qui correspondent à des niveaux distincts de forte densité du feuillage. Dans la régénération étudiée, la structure est monostrate à l'ombre et pluristrate en demi-lumière et à la lumière, avec respectivement 2 et 3 strates.

Seuls les plants sans dommages apparents ont été étudiés : 390 sur 403 . Les individus qui ont disparu avant 1986 n'ont évidemment pas été pris en compte. Cependant les densités élevées observées ici laissent supposer que leur nombre a été certainement faible parmi les grands plants, compétitifs, alors qu'il a pu être plus élevé parmi les plus petits, peu compétitifs. Nous pouvons done penser que le biais introduit par la disparition de certains individus doit être négligeable sur le développement morphologique des plants étudiés. Les effectifs analysés sous les 3 éclairements et dans les différentes strates ont été reportés sur le tableau $\mathrm{I}$.

\section{Analyse morphologique des individus}

Par définition, nous considérons qu'un axe est produit par un seul méristème terminal, depuis l'apparition de ce dernier jusqu'à son arrêt de fonctionnement. Tous les axes aériens du hêtre ont une croissance rythmique interannuelle qui se manifeste par la formation d'une pousse chaque année (Thiébaut et al, 1981).

Le développement des jeunes hêtres a été décrit en analysant uniquement leur tige principale et en considérant celle-ci comme un empilement de pousses annuelles (Thiébaut et al, 1985). Le long de la tige principale, les cicatrices laissées par le bourgeon terminal permettent de repérer exactement la base de toutes les pousses. La première pousse d'un individu est indiquée par les cicatrices opposées-décussées des 2 cotylédons et des 2 premiers bourgeons axillaires. La croissance de la tige principale a pu ainsi être reconstituée chaque année pendant 18 ans.

La description morphologique met l'accent sur 2 critères, la vigueur et la forme de la tige principale (Thiébaut, 1985). La vigueur a été analysée en mesurant la longueur des pousses annuelles et leur longueur cumulée chaque année (pour évaluer la "hauteur totale»), le nombre de ramifications de premier ordre dans chaque pousse et leur nombre total chaque année, le diamètre au collet et à la base de la dernière pousse en 1986. La forme a été décrite sur des individus de 18 ans, selon leur mode de ramification. À chaque ramification, l'importance de la dominance apicale et la préséance entre les bourgeons terminal et latéral ont une influence déterminante sur la forme de la tige principale :

- selon l'importance de la dominance, la tige est droite ou fourchue;

- selon la préséance entre les 2 bourgeons, la tige est monopodique ou sympodique.

La forme a été décrite à l'aide de ces 4 modes de ramifications, selon 2 points de vue : 
Tableau I. Caractères moyens de vigueur des plants à 18 ans selon l'éclairement et la strate. - Strates, légende commune aux tableaux : $L$, à la lumière, $\left(L_{1}\right)$ supérieure, $\left(L_{2}\right)$ intermédiaire et $\left(L_{3}\right)$ inférieure; I en demi-lumière, $\left(I_{1}\right)$ supérieure et $\left(I_{2}\right)$ inférieure; $\left(O_{1}\right)$ à l'ombre.

\begin{tabular}{|c|c|c|c|c|c|c|}
\hline & & & Éclairem & & & \\
\hline & & Lumière & & Demi-l & umière & Ombre \\
\hline Strates ${ }^{\circ}$ & $L_{1}$ & $L_{2}$ & $L_{3}$ & $I_{1}$ & $\mathrm{I}_{2}$ & $O_{1}$ \\
\hline Effectifs & 51 & 63 & 34 & 76 & 93 & 73 \\
\hline $\begin{array}{l}\text { Hauteur totale } \\
\text { (cm) }\end{array}$ & $181,4^{a}$ & $94,5^{b}$ & $36,3^{\theta}$ & $85,6 c$ & $45,2^{\alpha}$ & $31,4^{\theta}$ \\
\hline $\begin{array}{l}\text { Distribution } \\
p\left(\chi^{2}\right)\end{array}$ & $\begin{array}{c}\text { normale } \\
0,30\end{array}$ & $\begin{array}{c}\text { normale } \\
0,66\end{array}$ & $\begin{array}{c}\text { normale } \\
0,25\end{array}$ & $\begin{array}{l}\text { Poisson } \\
0,68\end{array}$ & $\begin{array}{c}\text { normale } \\
0,54\end{array}$ & $\begin{array}{c}\text { Poisson } \\
0,90\end{array}$ \\
\hline $\begin{array}{l}\text { Diamètre au collet } \\
(\mathrm{mm})\end{array}$ & $11,6^{a}$ & $8,2^{b}$ & $4,6^{d}$ & $6,6^{c}$ & $4,3^{d}$ & $3,7^{e}$ \\
\hline $\begin{array}{l}\text { Distribution } \\
p\left(\chi^{2}\right)\end{array}$ & $\begin{array}{l}\text { Poisson } \\
0,78\end{array}$ & $\begin{array}{l}\text { Poisson } \\
0,89\end{array}$ & $\begin{array}{l}\text { Poisson } \\
0,70\end{array}$ & $\begin{array}{l}\text { Poisson } \\
0,73\end{array}$ & $\begin{array}{c}\text { normale } \\
0,32\end{array}$ & $\begin{array}{l}\text { Poisson } \\
0,95\end{array}$ \\
\hline $\begin{array}{l}\text { Diamètre-86 } \\
(\mathrm{mm})\end{array}$ & $1,7^{a}$ & $1,5^{b}$ & $0,9^{\theta}$ & $1,3^{b}$ & $1,0^{d}$ & $1,1^{c}$ \\
\hline $\begin{array}{l}\text { Nombre de ramifications } \\
1^{\text {er }} \text { ordre }\end{array}$ & $49,9^{a}$ & $32,6^{b}$ & $14,2^{\mathrm{d}}$ & $25,9^{c}$ & $16,0^{d}$ & $8,9^{\circ}$ \\
\hline
\end{tabular}

* Test de Mann-Whitney, quand la lettre placée en exposant change, les valeurs sont significativement différentes entre elles au seuil de 5\%. $\mathrm{p} \chi^{2}$ : écart entre la distribution observée et la distribution théorique.

d'abord quantitatif, en dénombrant les effectifs de chaque type de ramification et ensuite, qualitatif, en dénombrant chaque type de tige. Dans ce dernier cas, on considère qu'une tige ayant au moins une fourche est "fourchue" et qu'une tige ayant une ramification sympodique est "sympodique". De ce fait, les tiges droites n'ont aucune fourche et les tiges monopodiques aucune ramification sympodique.

Parmi les caractères de vigueur, la longueur, la hauteur totale et le nombre de ramifications sont stables et acquis définitivement à la fin de la période de végétation, alors que le diamètre au collet et les criteres de forme peuvent varier dans le temps. En conséquence, la reconstitution de la croissance du plant ne portera que sur la longueur des pousses, la hauteur totale et le nombre de ramifications. La croissance de la tige en diamètre n'a pas été reconstituée pour éviter une destruction des plants.

\section{RÉSULTATS}

\section{Morphologie des plants à l'âge de 18 ans}

La distribution des hauteurs présente 3 modes, correspondant à 3 strates à la lu- 
mière (de haut en bas : $L_{1}, L_{2}$ et $L_{3}$ ), 2 en demi-lumière (de haut en bas : $l_{1}$ et $l_{2}$ ), et 1 seul à l'ombre $\left(O_{1}\right)$, tableau I. Sous chaque éclairement, les plants sont presque aussi nombreux d'une strate à l'autre, sauf dans $L_{3}$ ). La distribution des hauteurs peut être ajustée à une courbe théorique normale dans chaque strate à la lumière et dans $\left(I_{2}\right)$, à une courbe de Poisson dans $\left(I_{1}\right)$ et dans $\left(O_{1}\right)$. Cependant, ces ajustements sont de valeurs inégales car la probabilité du $\chi^{2}$ varie entre 0,25 et 0,90 . La distribution des diamètres au collet ne change guère et reste conforme à une courbe de Poisson, sauf dans $\left(\mathrm{I}_{2}\right)$. Les ajustements sont ici plus réguliers et meilleurs que précédemment, sauf dans $\left(l_{2}\right)$.

Les hauteurs et les diamètres au collet sont corrélés. À la lumière, les individus forment 3 nuages de points, correspondant aux 3 strates, figure 1. L'amplitude de va- riation de la hauteur est bien définie à l'intérieur de chaque strate, alors que le diamètre varie de manière continue entre les strates; en sorte que les individus se distinguent surtout en fonction de leur hauteur, d'une strate à l'autre, et selon leur diamètre à l'intérieur d'une strate. Une relation asymptotique lie la hauteur au diamètre dans chaque strate. Les corrélations de Spearman sont positives, significatives, faibles dans $\left(L_{1}\right),\left(L_{2}\right)$ et forte dans $\left(L_{3}\right)$. En demi-lumière, les individus ne forment plus qu'un nuage de points, même s'il est encore possible de distinguer 2 strates (fig 2). La hauteur et le diamètre varient de manière continue dans la régénération et sont liés par une relation quasi linéaire. Les corrélations sont positives, significatives et plus élevées qu'à la lumière. A l'ombre, à part quelques sujets vigoureux, aucune stratification n'apparaît (fig 3). La

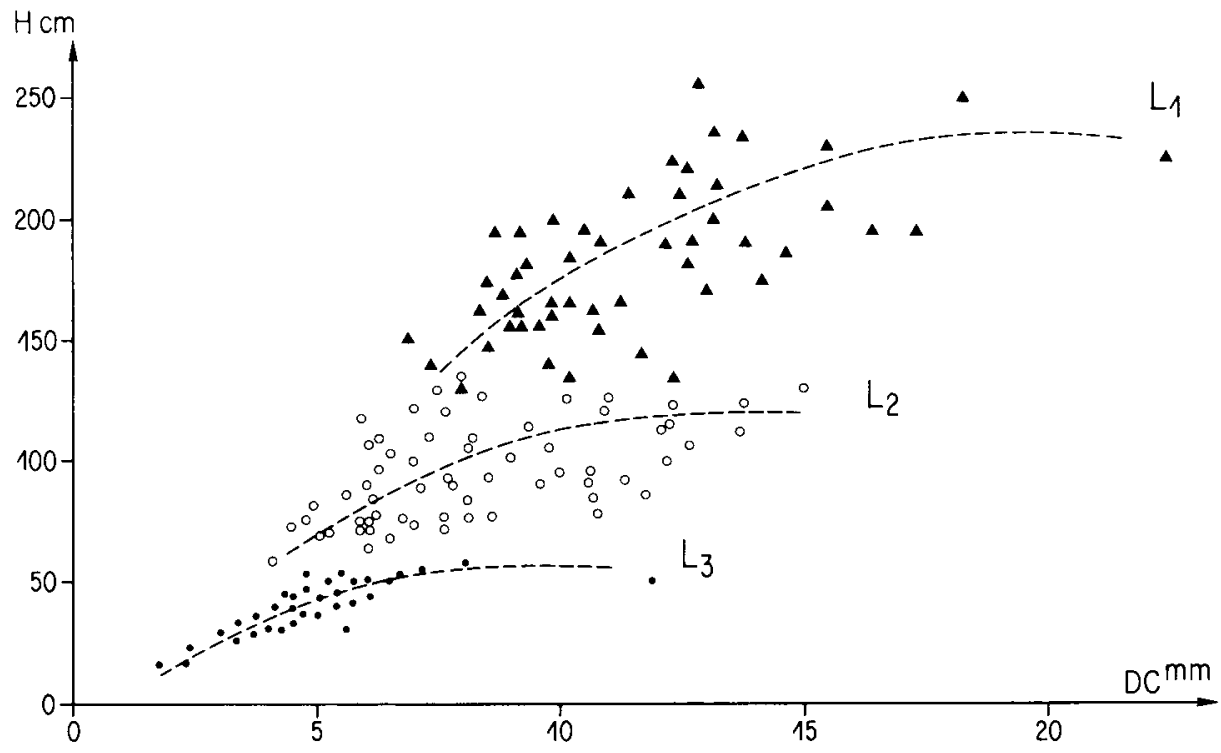

Fig 1. Hauteur totale $(H)$ et diamètre au collet (DC) à la lumière à 18 ans. Pour la totalité de la régénération : $N=148$ et $r s$ (coefficient de Spearman) $=0,73^{* *},\left(\mathrm{~L}_{1}\right):$ strate supérieure, $n_{1}=51$ et $r s_{1}=$ $0,57^{\star * *} ;\left(L_{2}\right)$ : strate intermédiaire, $n_{2}=63$ et $r s_{2}=0,57^{\star \star *} ;\left(L_{3}\right)$ : strate inférieure, $n_{3}=34$ et $r s_{3}=$ $0,81^{* * *}$. 


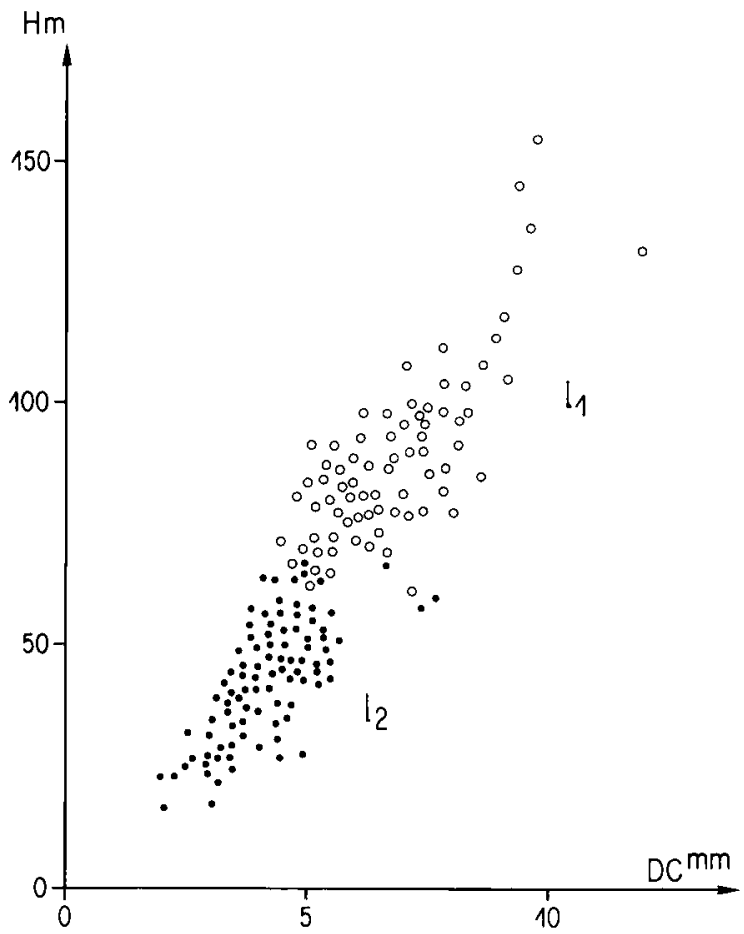

Fig 2. Hauteur totale $(H)$ et diamètre au collet $(D C)$ en demi-lumière à 18 ans. Pour la totalité de la régénération : $N=169$ et $r s$ (coefficient de Spearman) $=0,82^{* * *},\left(I_{1}\right):$ strate supérieure, $n_{1}=76$ et $r s_{q}=$ $0,63^{* * \star},\left(l_{2}\right)$ : strate inférieure, $n_{2}=93$ et $r s_{2}=0,73^{* * *}$.

corrélation entre hauteur et diamètre est quasi linéaire, positive et significative.

La densité varie d'un éclairement à l'autre. Le nombre moyen d'individus par $m$ linéaire est de : 15,9 à la lumière, 22,4 en demi-lumière et 8,9 à l'ombre. Les écarts sont significatifs entre ces 3 valeurs prises 2 à 2. Une interaction densitééclairement n'est pas exclue et peut avoir une influence sur la croissance des plants. L'influence de la densité a été appréciée en calculant les corrélations de Spearman entre la densité et 8 caractères morphologiques, dont 4 pour la vigueur (hauteur totale, diamètre au collet, diamètre-86, nombre de ramifications primaires) et 4 pour la forme (nombre de ramifications monopodiques, sympodiques, droites et fourchues). La plupart des coefficients ne sont pas significatifs, sauf pour les diamètres dans $\left(L_{2}\right)$, et presque toutes les valeurs sont faibles, inférieures à 0,30 . Dans cette régénération, les variations de la densité ont donc peu d'influence sur le développement des jeunes hêtres, sans doute en raison de la valeur excessive de la densité. Nous ne tiendrons plus compte des variations de celle-ci par la suite.

La vigueur et la forme des plants varient en fonction de l'éclairement et de la strate 


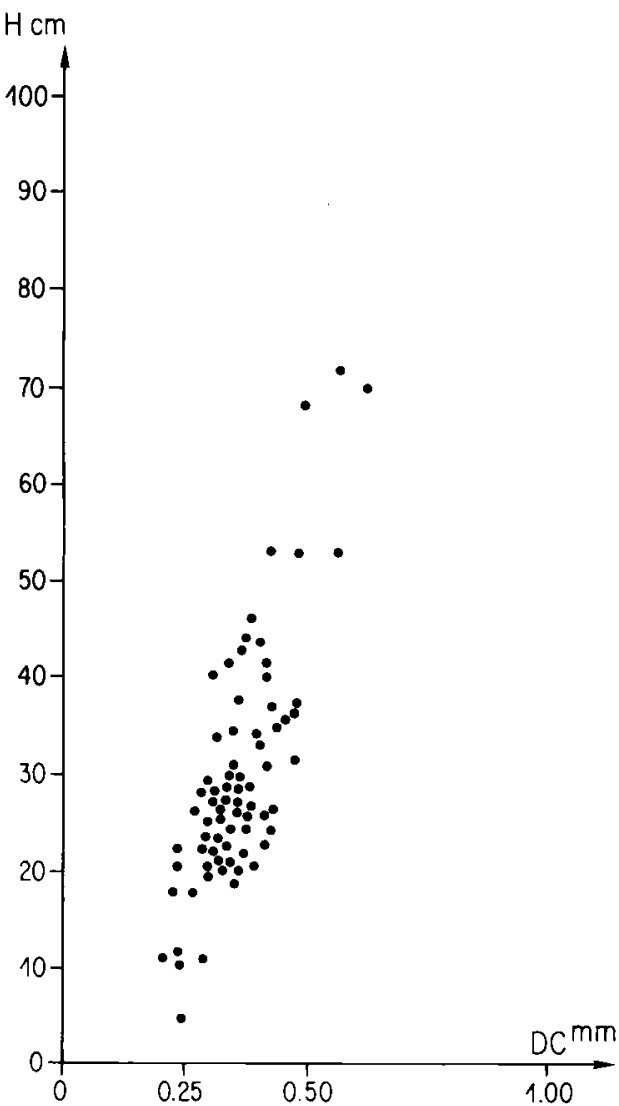

Fig 3. Hauteur totale $(\mathrm{H})$ et diamètre au collet (DC) à l'ombre à 18 ans. Pour la totalité de la régénération : $N=73$ et rs (coefficient de Spear$\operatorname{man})=0,67^{* *}$.

selon 2 gradients de luminosité. Lorsque la luminosité augmente, dans des strates analogues en allant de l'ombre vers la lumière et, sous un éclairement, en allant de la strate inférieure vers la supérieure :

- la vigueur augmente significativement par la hauteur totale, le diamètre au collet, le diamètre-86 et le nombre de ramifications de premier ordre, tableau I. Les écarts entre les strates sont plus grands à la lumière,
- la forme change car le nombre de ramifications droites et monopodiques augmente de manière significative, tableau II. Le nombre de fourches et le pourcentage de tiges fourchues augmentent aussi alors que le pourcentage de ramifications fourchues et le nombre de ramifications sympodiques diminuent, mais les écarts ne sont pas toujours significatifs (tableaux II, III).

Une analyse discriminante a été réalisée pour tester les écarts entre les plants selon l'éclairement et selon leur strate ( 3 à la lumière +2 en demi-lumière +1 à l'ombre $=6$ groupes), en utilisant 4 variables de vigueur : la hauteur totale, le diamètre au collet, le diamètre-86, le nombre de ramifications, et 2 variables de forme : le nombre de ramifications droites et monopodiques. Les ramifications fourchues et sympodiques n'ont pas été retenues car ces caractères sont alternatifs avec les précédents. La discrimination est totale entre les individus des 3 strates à la lumière, mais elle n'est plus complète entre les individus des 2 strates en demi-lumière (fig 4). Sur l'ensemble du transect, seuls les individus de la strate supérieure à la lumière se distinguent de tous les autres.

\section{Croissance des plants au cours des 18 premières années}

La croissance annuelle, caractérisée par la longueur de la pousse et le nombre de ramifications de premier ordre, se différencie, très tôt d'une strate à l'autre sous le même éclairement : dès la seconde année en demi-lumière et entre la seconde et la quatrième année à la lumière (figs 5 et 6 ).

Ainsi, avant 1971 et même jusqu'en 1973, la croissance reste comparable sous les 3 éclairements et dans les différentes strates, même si certains écarts sont déjà significatifs. Après 1973, la croissance annuelle diffère davantage selon l'éclaire- 
Tableau II. Caractères moyens de forme des plants à 18 ans, selon l'éclairement et leur strate.

\begin{tabular}{|c|c|c|c|c|c|c|}
\hline \multirow[t]{2}{*}{ Eclairements } & \multirow[t]{2}{*}{ Strates } & \multicolumn{4}{|c|}{ Nombre de ramifications de premier ordre } & \multirow{2}{*}{$\frac{\text { Nombre de fourches }}{\text { Nombre de ramifications }}$} \\
\hline & & Droites & Fourchues & Monopod & Sympod & \\
\hline & $\begin{array}{l}L_{1} \\
n=51\end{array}$ & $49,5^{a}$ & $0,4^{a}$ & $49,6^{a}$ & $0,4^{a}$ & $0,8^{a}$ \\
\hline \multirow[t]{2}{*}{$\begin{array}{l}\text { Lumière } \\
n=148\end{array}$} & $\begin{array}{l}L_{2} \\
n=63\end{array}$ & $32,3^{b}$ & $0,3^{a}$ & $32,2^{b}$ & $0,4^{a}$ & $1,1^{a}$ \\
\hline & $\begin{array}{l}L_{3} \\
n=34\end{array}$ & $14,0^{d}$ & $0,2^{b}$ & $14,0^{\mathrm{d}}$ & $0,7^{b}$ & $1,9 \mathrm{ab}$ \\
\hline $\begin{array}{l}\text { Demi- } \\
\text { lumière }\end{array}$ & $\begin{array}{l}l_{1} \\
n=76\end{array}$ & $25,2^{c}$ & $0,4^{a}$ & $25,4^{c}$ & $0,5^{a}$ & $1,7 \mathrm{ab}$ \\
\hline$n=169$ & $\begin{array}{l}l_{2} \\
n=93\end{array}$ & $15,7^{d}$ & $0,3^{a b}$ & $14,7^{d}$ & $0,5^{a}$ & $2,5^{b}$ \\
\hline $\begin{array}{l}\text { Ombre } \\
n=73\end{array}$ & $O_{1}$ & $8,5^{\ominus}$ & $0,4^{a}$ & $8,3^{\theta}$ & $0,6^{b}$ & $6,6^{c}$ \\
\hline
\end{tabular}

* Test de Mann-Whitney, quand la lettre placée en exposant change, les valeurs sont significativement différentes entre elles au seuil de $5 \%$.

ment et la strate. Elle est significativement plus grande dans $\left(L_{1}\right)$ que dans toutes les autres strates. Elle reste comparable dans $\left(L_{2}\right)$ et dans $\left(l_{1}\right)$, entre lesquelles les écarts ne sont pas significatifs; ainsi que dans $\left(L_{3}\right),\left(I_{2}\right)$ et $\left(O_{1}\right)$. À la fin de la période étudiée, après 1983, la croissance annuelle diminue et les écarts entre éclairements et strates s'atténuent. Ainsi on peut distinguer 3 phases de croissance, mieux marquées à la lumière qu'en demi-lumière : une phase d'installation jusqu'à l'âge de 5 ans pendant laquelle les courbes sont régulières et ont à peu près la même allure, une phase de différenciation entre 5 et 15 ans pendant laquelle les courbes divergent selon l'éclairement et la strate et pré- sentent des oscillations interannuelles importantes, enfin une phase de régression entre 15 et 18 ans pendant laquelle les courbes redeviennent régulières et se rapprochent les unes des autres. Aucune évolution comparable à celle-ci n'apparaît à l'ombre où la croissance reste toujours faible. Des analyses discriminantes confirment l'existence de ces 3 phases. La croissance annuelle a été comparée selon l'éclairement et selon la strate $(=6$ groupes), en utilisant la longueur de la pousse annuelle et le nombre de ramifications de premier ordre (2 variables quantitatives), (fig 7). Les individus ne se distinguent guère au début (en 1969) et à la fin (en 1985) de la période considérée, quels 
Tableau III. Types de tiges principales à 18 ans (en \%) selon leur mode de ramification en fonction de l'éclairement et de leur strate.

Eclairements et strates Droites Fourchues $\chi^{2}$ Monopodiques Sympodiques $\chi^{2}$

Lumière

$n=148$

$L_{1}$

$n=51$

$\mathrm{L}_{2}$

$n=63$

$\mathrm{L}_{3}$

$n=34$

Dermi-lumière

$n=169$

$l_{1}$

$n=76$

$\stackrel{\mathrm{I}_{2}}{n=93}$

Ombre $O_{1}$

$n=73$
66,

$74,6 \quad 25,4$

76,5

23,5

35,3

70,6

29,4

(a)

63,5

36,5

(a)

64,7

(b)

$$
53,5
$$

$81,1 \quad 18,9$

(a)

58,6

56,3

43,7

(a)

$\chi^{2}$ : test $\chi^{2}$ sur les effectils, quand la lettre placée en exposant change les valeurs sont significativement differentes entre elles au seuil de $5 \%$.

que soient leur éclairement et leur strate, alors qu'ils se différencient davantage au milieu de cette période (en 1983).

Avant 1973, le développement est comparable dans toute la régénération par la hauteur totale (fig 8 ), et le nombre de ramifications de premier ordre, (fig 9). Après 1973, il diffère progressivement et de manière significative selon l'éclairement et selon la strate. II est de plus en plus grand dans $\left(L_{1}\right)$. Il progresse moins vite et reste du même ordre de grandeur dans $\left(L_{2}\right)$ et dans $\left(I_{1}\right)$. Enfin, il augmente peu dans $\left(L_{3}\right)$, $\left(l_{2}\right)$ et $\left(O_{1}\right)$, entre lesquelles le développement reste comparable. À la fin de la période considérée, après 1983 , les écarts se stabilisent entre toutes ces situations. On peut donc distinguer 3 phases de développement dans la régénération : d'installation, de différenciation et de stabilisation. La distribution des hauteurs est toujours unimodale pendant la première phase, puis elle devient plurimodale au cours de la seconde à la lumière et en demilumière. Des analyses discriminantes ont été réalisées chaque année pour comparer 


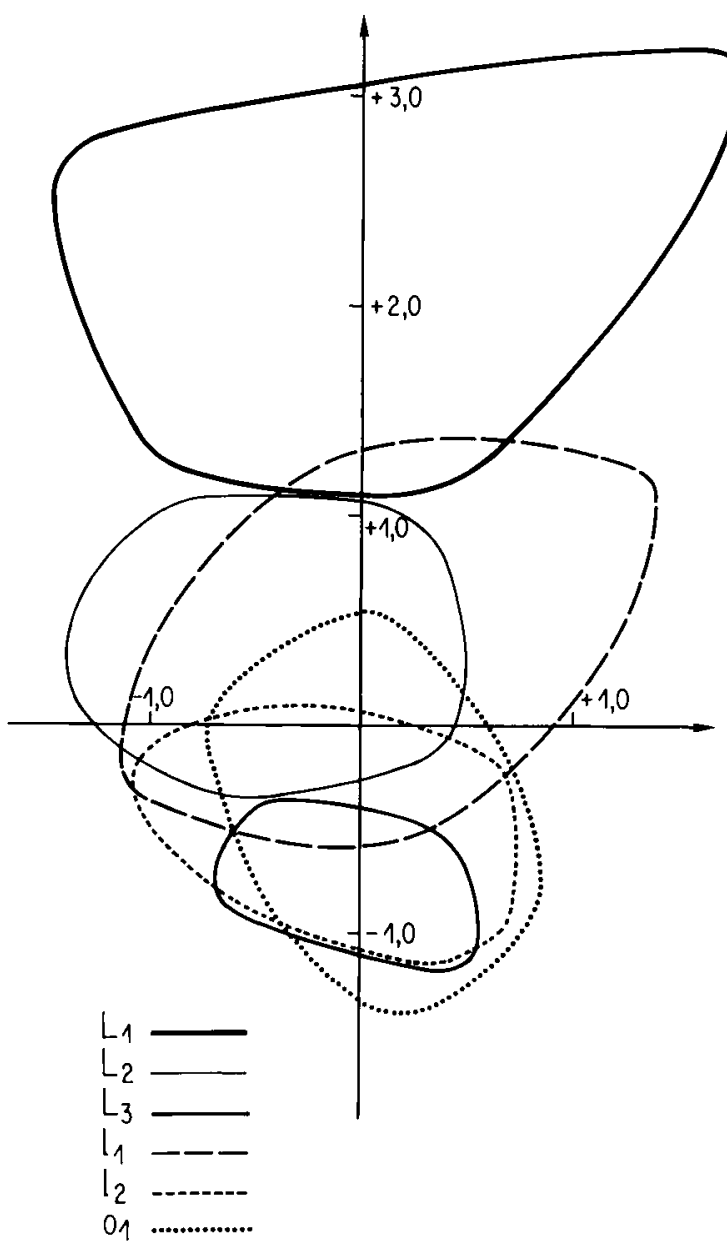

Fig 4. Analyse discriminante de tous les individus sur 6 critères de forme et de vigueur à 18 ans, selon l'éclairement et leur strate. Métrique des individus : $N=390$, dont 51 dans $\left(L_{1}\right), 63$ dans $\left(L_{2}\right)$ et 34 dans $\left(L_{3}\right) ; 76$ dans $\left(I_{1}\right)$ et 93 dans $\left(\mathrm{I}_{2}\right) ; 73$ dans $\left(\mathrm{O}_{1}\right)$. Tous les individus ont le même poids. Métrique des variables : 6 variables quantitatives avec la hauteur totale, le diamètre au collet, le diamètre-86, le nombre de ramification de $1^{\text {er }}$ ordre, le nombre de ramifications monopodiques et droites; 1 variable qualitative selon la strate et l'éclairement : 6 groupes. Inertie absorbée, axe $1: 74,40 \%$ et axe II : $18,47 \%$. le développement selon l'éclairement et selon la strate ( $=6$ groupes), en utilisant 2 variables quantitatives : la hauteur totale et le nombre total de ramifications de premier ordre (fig 10). Ces analyses confirment la différenciation progressive des individus selon l'éclairement et selon leur strate, ainsi que l'existence de ces 3 phases. A la lumière, les strates inférieure et supérieure, sont complètement disjointes à partir de 1979. En demi-lumière, la discrimination n'est jamais totale entre les 2 strates. Et après 1983, l'écart se maintient entre les éclairements et les strates malgré le ralentissement de la croissance annuelle.

\section{DISCUSSIONS}

\section{Morphologie des plants âgés de 18 ans}

Au Lingas, les hauteurs et les diamètres observés à l'âge de 18 ans sont faibles, comparés à ceux fournis par d'autres auteurs pour des hêtres du même âge (Polge, 1983; Falcone et al, 1986; Lanier, 1986). Ce faible développement peut avoir plusieurs causes : le climat rigoureux de montagne à l'Aigoual, la densité élevée de la régénération, la médiocrité du sol acide.

Certains auteurs admettent que dans un peuplement pur, équien et clair, où la compétition est faible à nulle, la distribution des hauteurs et des diamètres est normale (Prodan, 1953, Toda, 1963; Delvaux, 1966). Dans cette situation la structure est monostrate (Delvaux, 1966 et Falcone, 1985). Par contre, lorsque le peuplement se ferme, la compétition augmente et se manifeste par des vigueurs individuelles différentes. Chez les espèces intolérantes à l'ombre, la structure demeure monostrate (Molher et al, 1978) alors que chez les tolérantes, comme le hêtre, la structure peut devenir pluristrate (Ford, 1975). En effet, 


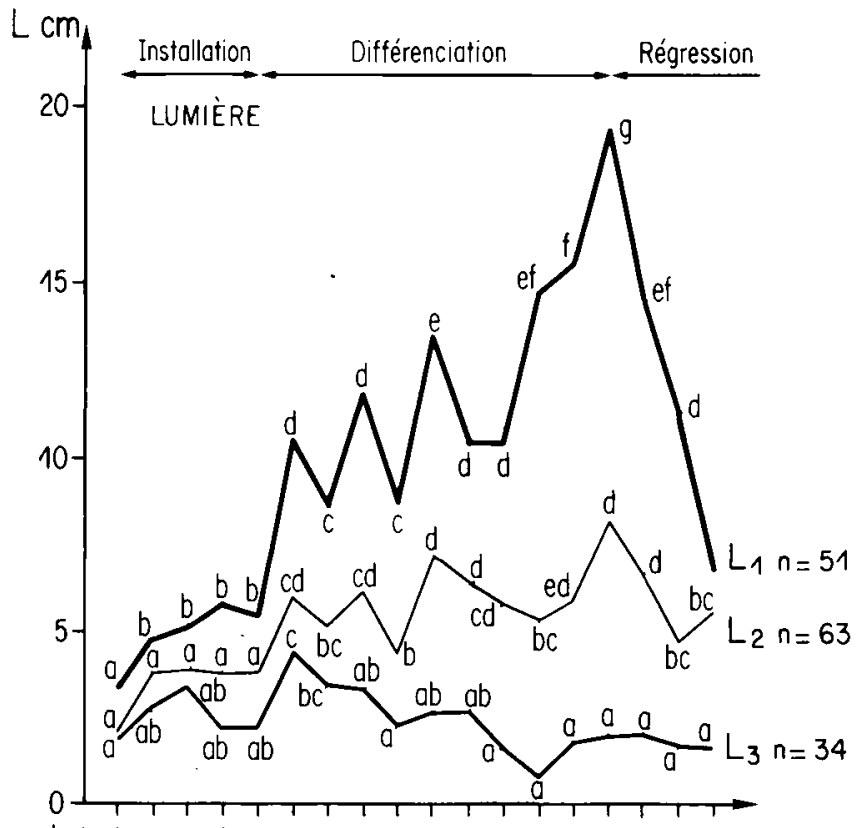

L1 A A A A A A A A A A A A A A A A A A

L2 A $B$ B $B$ B $B$ B $B$ B $B$ B $B$ B $B$ B $B$ B $B$ B $B$ B $B$ B

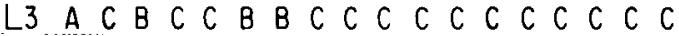
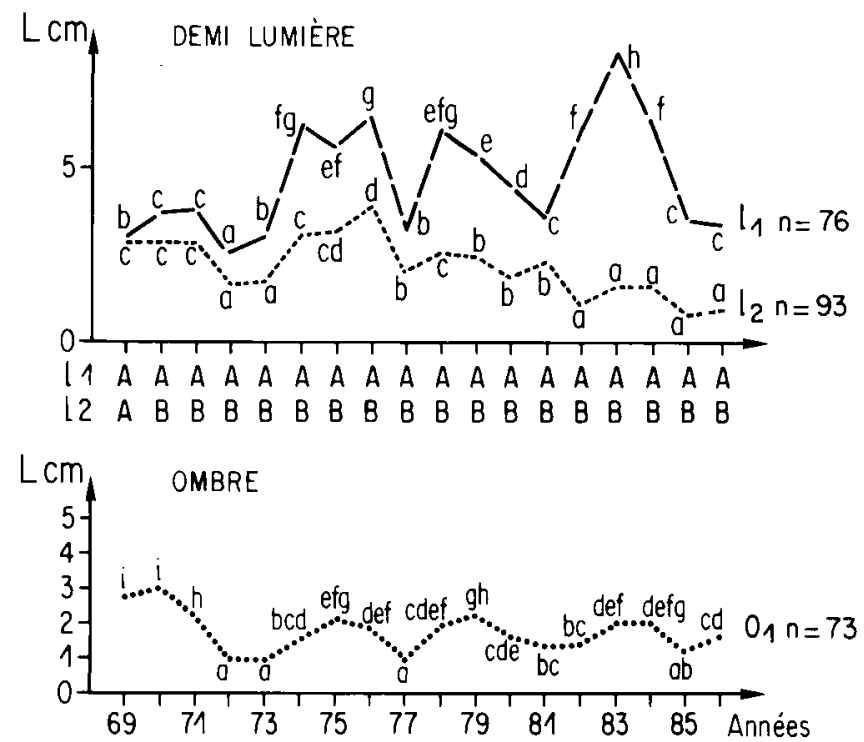

Fig 5. Croissance annuelle, longueur moyenne des pousses $(L)$ pendant les 18 premières années. Les valeurs sont différentes au seuil de 5\% (test de Mann-Whitney) : - dans une strate, entre les années quand la lettre minuscule change; - sous le même éclairement ef dans la même année, entre les strates quand la lettre majuscule change. 

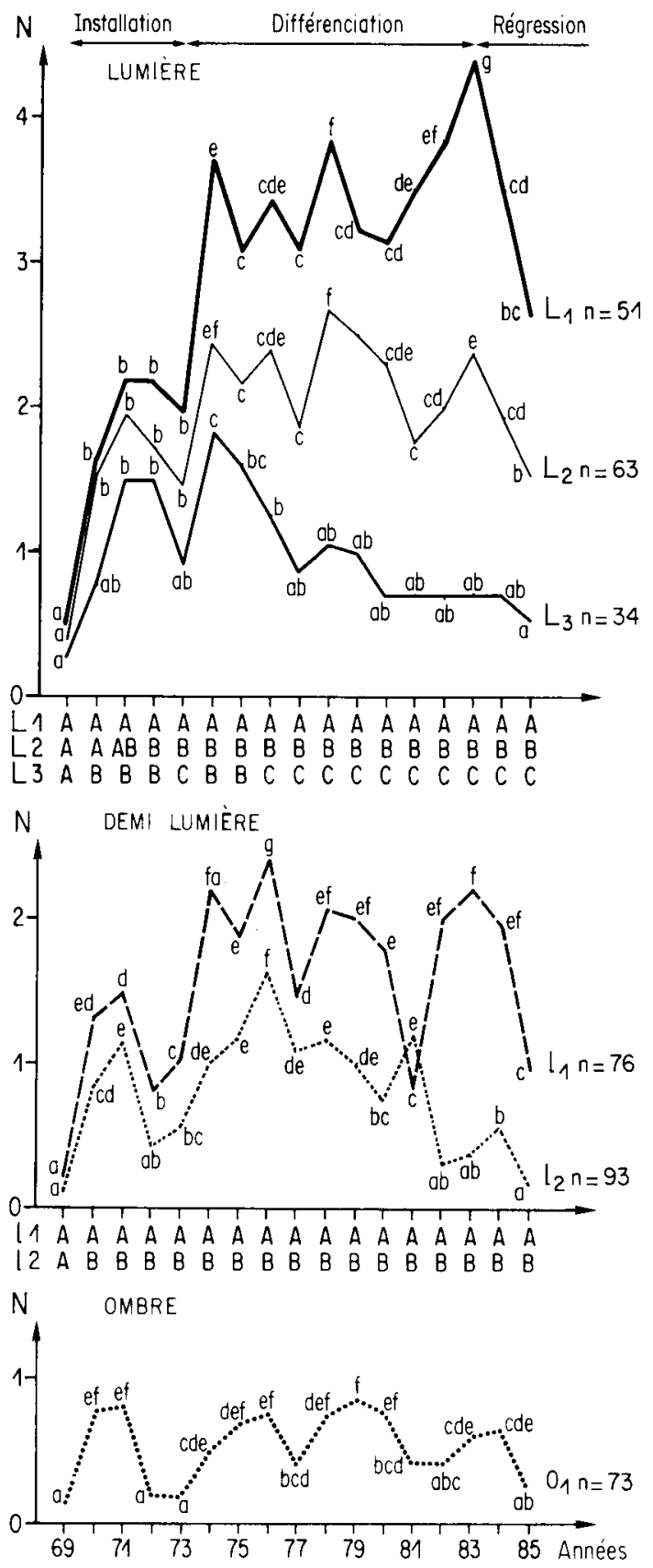

Fig 6. Croissance annuelle, nombre moyen de ramifications de premier ordre (N) pendant les 18 premières années. Les valeurs sont différentes au seuil de 5\% (test de Mann-Whitney) : - dans une strate, entre les années quand la lettre minuscule change; - sous le même éclairement et dans la même année, entre les strates quand la lettre majuscule change. 


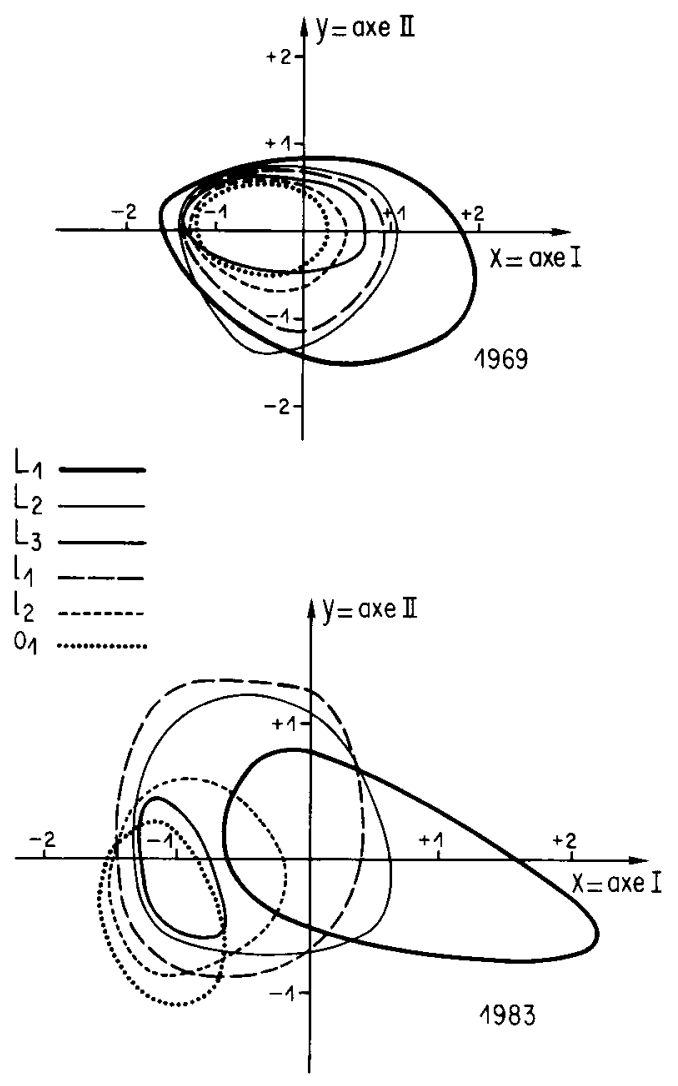

malgré des conditions médiocres de luminosité, des plants plus petits peuvent se maintenir et former une ou plusieurs strates subordonnées sous la strate principale. La distribution des hauteurs devient alors plurimodale. Au Lingas, ce deuxième cas a été observé à la lumière et en demilumière mais pas à l'ombre où la structure reste monostrate malgré une densité élevée ( 9 sujets en moyenne par $m$ linéaire), tableau I. Donc, si l'absence de compétition entraîne ipso facto celle d'une stratification, une compétition, si forte soit-elle, ne provoque pas obligatoirement l'apparition d'une structure pluristrate, même chez une espèce tolérante. Compétition et stratification doivent être clairement distinguées.

Deux explications peuvent être avancées pour comprendre l'absence de stratification à l'ombre :

- tous les individus ont des compétences médiocres et se développent mal,

- le milieu est trop contraignant et écrase les differences de vigueur individuelle. Deux arguments plaident en faveur de cette seconde hypothèse. D'abord, le développement des individus situés à l'ombre est faible comparé à celui des autres plants et souligne ainsi les conditions limites de la luminosité sous le couvert. Par

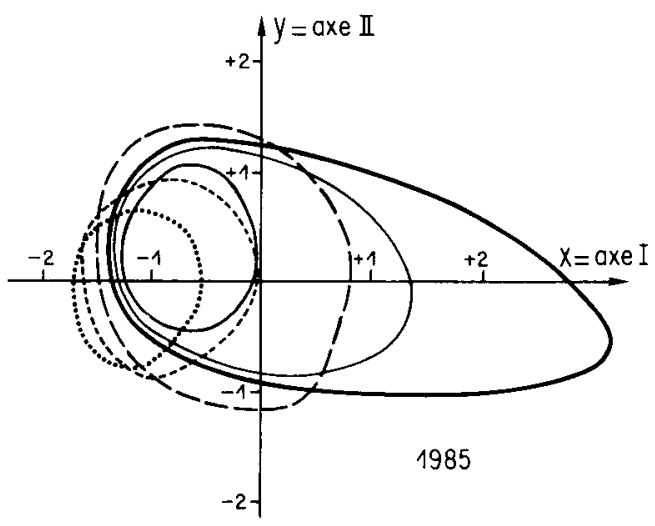

Fig 7. Analyses discriminantes sur la croissance annuelle pendant les 18 premières années. Métrique des individus : $N=390$, dont 51 $\left(L_{1}\right), 63$ dans $\left(L_{2}\right)$ et 34 dans $\left(L_{3}\right) ; 76$ dans $\left(l_{1}\right)$ et 93 dans $\left(\mathrm{I}_{2}\right) ; 73$ dans $\left(\mathrm{O}_{1}\right)$. Tous les individus ont le même poids. Métrique des variables : 2 variables quantitatives avec la longueur des pousses et le nombre de ramifications de ter $^{\text {r }}$ ordre, 1 variable qualitative selon la strate d'éclairement : 6 groupes. Interties absorbées en 1969 , axe I : 82,5\% et axe II : $10,1 \%$, en 1983 , axe $1: 73,6 \%$ et axe $\|: 6,7 \%$, en 1985 , axe I: $79,2 \%$ et axe II : $8,1 \%$. 

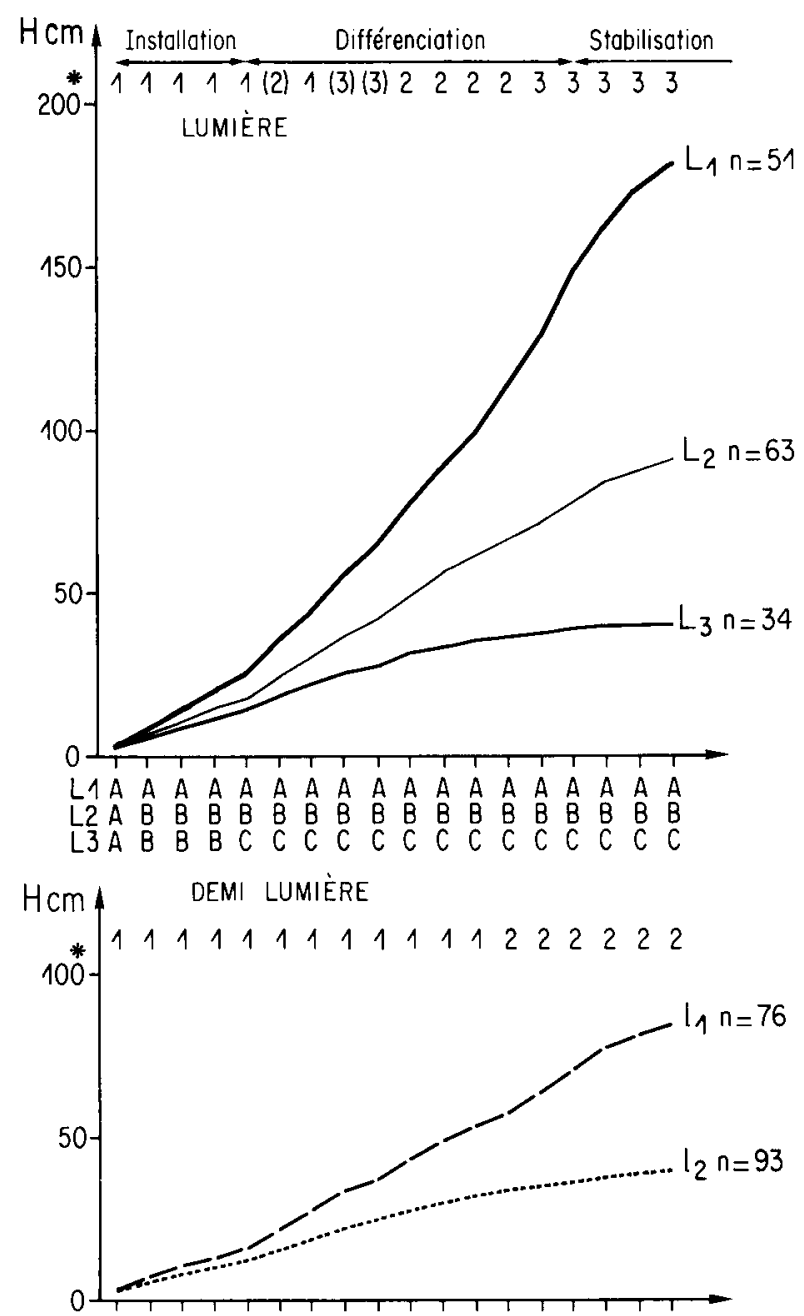

11 A A A A A A A A A A A A A A A A A A

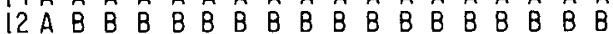

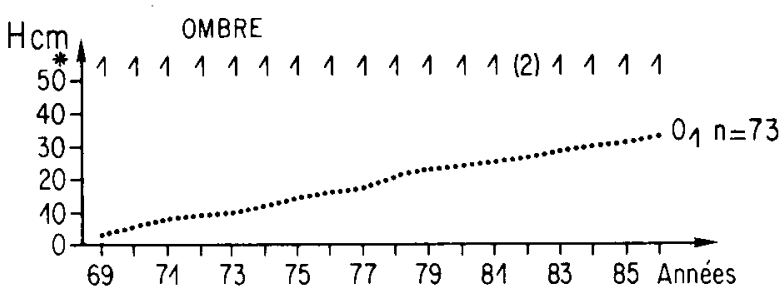

Fig 8. Croissance cumulée pendant les 18 premières années, hauteur totale moyenne $(H)$. Les valeurs sont différentes au seuil de $5 \%$ sous le même éclairement et dans la même année entre les strates quand la lettre majuscule change (test de Mann-Whitney). * Nombre de modes observés dans la distribution de toutes les hauteurs au cours d'une année. 

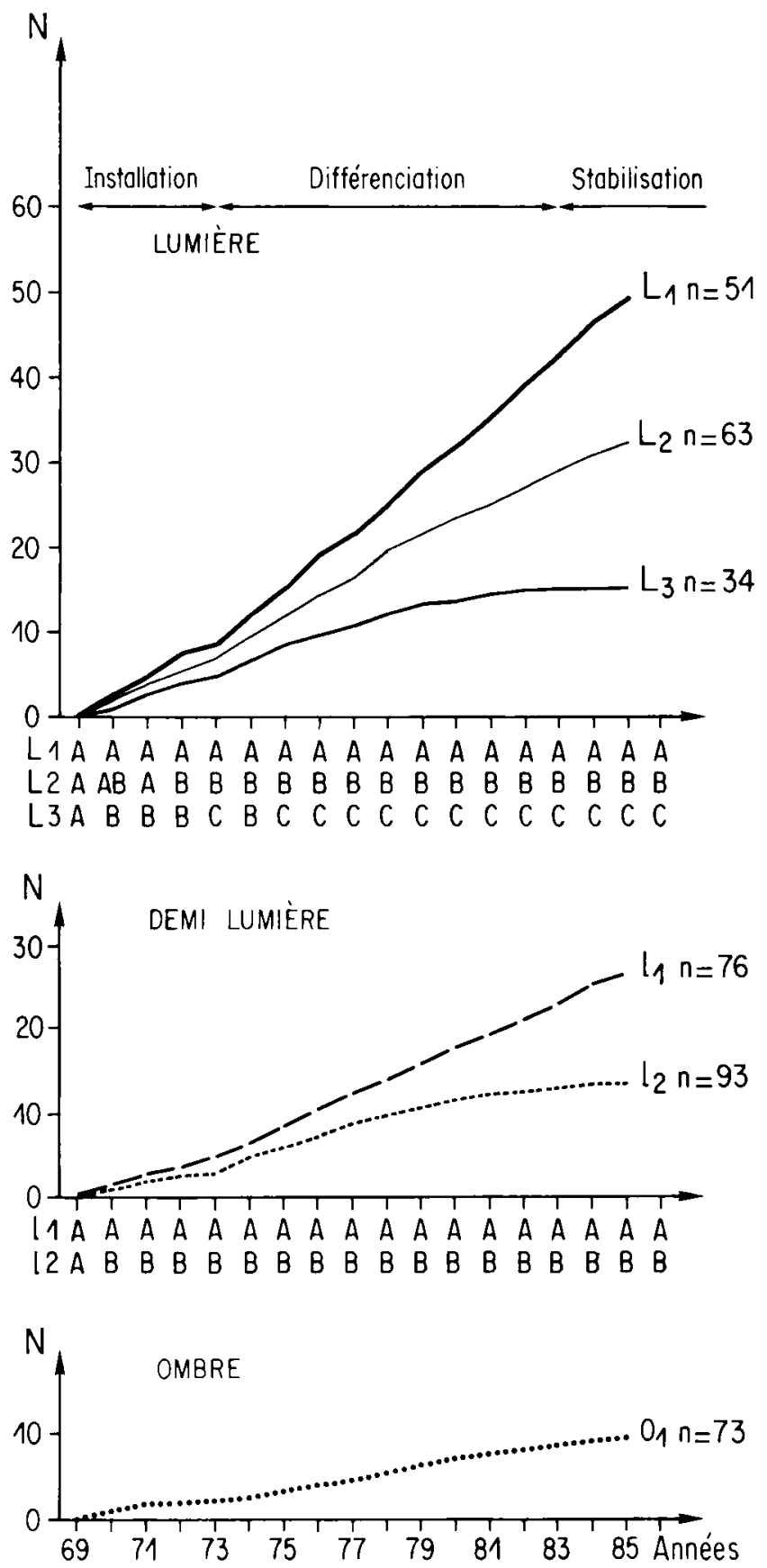

Fig 9. Croissance cumulée pendant les 18 premières années, nombre total moyen de ramifications de premier ordre $(\mathrm{N})$. Les valeurs sont différentes au seuil de $5 \%$ sous le même éclairement et dans la même année entre les strates quand la lettre majuscule change (test de Mann-Whitney). 


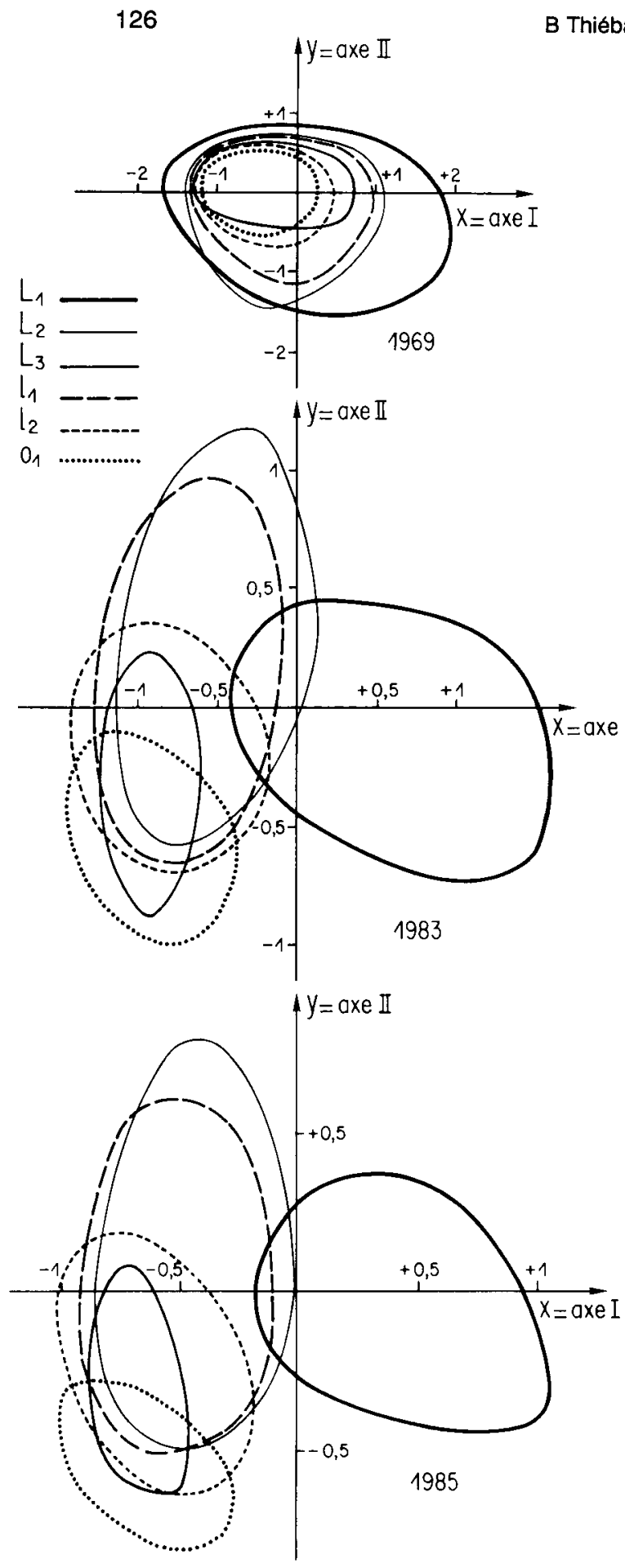

ailleurs, l'étude de la diversité génétique dans cette régénération à l'aide de 6 loci ne révèle aucune différence significative du polymorphisme génétique entre les 3 éclairements (Comps et al, 1988; Thiébaut et Comps, 1992).

Dans une régénération dense, monostrate ou pluristrate, les distributions de hauteurs et de diamètres peuvent être symétriques ou dissymétriques dans chaque strate (Bliss et Reinker, 1964; Ford et Newbould, 1970). Par rapport à une distribution normale, Delvaux (1966) considère qu'une asymétrie à gauche révèle une situation de disète et une asymétrie à droite, a une situation d'abondance. Plusieurs études montrent que les peuplements issus de graines passent par 3 périodes au début de leur développement :

- après germination, la compétition est faible et les distributions de hauteurs et de diamètres sont normales;

- pendant la croissance, la compétition augmente et les distributions deviennent dissymétriques à gauche;

- puis au-delà d'un seuil de tolérance (selfthinning line) dû à une compétition excessive, on observe la mort des petits individus et la normalisation des distributions (Hough, 1932; Schnur, 1934; Koyama et Kira, 1956; Black et Wilkinson, 1963; Yoda et al, 1963; Lee, 1971; Johnson, 1975;

Fig 10. Analyses discriminantes sur la croissance cumulée pendant les 18 premières années. Métrique des individus : $N=390$, dont 51 dans $\left(L_{1}\right), 63$ dans $\left(L_{2}\right)$ et 34 dans $\left(L_{3}\right) ; 76$ dans $\left(I_{1}\right)$ et 93 dans $\left(l_{2}\right) ; 73$ dans $\left(O_{1}\right)$. Tous les individus ont le même poids. Métrique des variables : 2 variables quantitatives avec la hauteur totale et le nombre de ramifications de 1er ordre, 1 variable qualitative selon la strate de l'éclairement : 6 groupes. Inerties absorbée, en 1969, axe $1: 82,5 \%$ et axe II : $10,1 \%$, en 1983 , axe I: $70,2 \%$ et axe $11: 10,5 \%$, en 1985 , axe $1: 68,7 \%$ et axe $\|: 8,3 \%$. 
Ford, 1975; Molher et al, 1978). Au Lingas, nous avons observé des distributions symétriques et asymétriques pour les hauteurs et les diamètres, tableau I, ce qui peut traduire une compétition élevée et annoncer une prochaine réduction du nombre de tiges. Mais les distributions de hauteurs et de diamètres sont différentes dans chaque strate à la lumière alors qu'elles sont identiques dans chaque strate en demi-lumière et à l'ombre. Donc, à la lumière, les variations de hauteur et de diamètre ne traduisent pas tout à fait la même réalité :

- soit parce que le diamètre est plus sensible que la hauteur à la compétition et présente de ce fait une dissymétrie plus précoce. Dans ce cas, les écarts entre la lumière et les autres éclairements seraient liés à une fermeture du peuplement, plus rapide à la lumière;

- soit parce que la hauteur est régularisée par la stratification ef non le diamètre. Dans ce cas, les écarts observés relèveraient d'une différence fondamentale dans le fonctionnement de la régénération.

À la lumière et en demi-lumière les variations de vigueur s'organisent en 2 composantes, interstrate et intrastrate. Les écarts interstrates sont supérieurs aux écarts intrastrates à la lumière, ce qui n'est plus le cas en demi-lumière (fig 4). II s'agirait là d'une différence de degré de la stratification, celle-ci étant plus évoluée à la lumière qu'en demi-lumière. Mais, les variations inter- et intrastrate portent respectivement sur des différences de hauteur et de diamètre à la lumière, alors qu'en demi-lumière ces 2 composantes se manifestent simultanément par des différences de hauteur et de diamètre (figs 1 et 2). En conséquence, les distributions de hauteurs et de diamètres ont bien des significations distinctes et peuvent être différentes dans une strate à la lumière, alors qu'elles expriment la même réalité et sont identiques dans une strate en demi- lumière, tableau I. De manière plus fondamentale, le fonctionnement de la régénération paraît distinct entre ces 2 éclairements.

La forme varie de manière plus discrète et plus complexe. En effet, si les variations selon l'éclairement et selon la strate sont simples et significatives pour les ramifications droites et monopodiques, ce n'est plus le cas pour les ramifications fourchues, tableaux III et IV. Dans une structure pluristrate, en allant du bas vers le haut, le nombre de fourches et le pourcentage de tiges fourchues augmentent avec la luminosité alors que le pourcentage de ramifications fourchues diminue. Ces résultats apparemment contradictoires traduisent 2 phénomènes distincts. Dans le premier cas, la fourchaison serait en relation avec la plus grande vigueur des plants dans la strate supérieure bien éclairée (Falcone, 1985; Dupré, 1984; Dupré et al, 1986) alors que dans le second cas, elle serait en relation avec une accommodation des plants à l'ombre dans les strates inférieures où ils ont tendance à former des houppiers “en éventail» (Thiébaut et al, 1985; Thiébaut, 1986). Le nombre de ramifications et de tiges sympodiques augmente de haut en bas, lorsque la luminosité diminue, et ce mode de ramification favorise l'édification de houppier en éventail. Ainsi la stratification a bien une influence sur les modalités de croissance des individus.

\section{Croissance des plants au cours des 18 premières années}

Les résultats obtenus reposent sur un a priori puisque les plants ont été classés de manière définitive dans la strate à laquelle ils appartenaient en 1986, sans tenir compte des reclassements éventuels pendant les années précédentes. Ces change- 
ments seront examinés dans un deuxième article.

Les croissances, annuelle et cumulée, varient selon l'éclairement et la strate dès les premières années (figs 5-9). Des résultats comparables ont été obtenus en pépinière par Gauthier (1987). À la lumière et en demi-lumière, le développement présente 3 phases qui sont absentes à l'ombre où la luminosité est un facteur limitant. Après une phase d'installation, puis de différenciation, pendant lesquelles la croissance augmente, sa diminution à la fin de la période étudiée, pendant la phase de stabilisation, est certainement liée à la densité excessive de la régénération et à la fermeture du couvert. Les 2 premières phases semblent correspondre aux 2 premières périodes décrites par Molher et al (1978). Mais au Lingas, une nouvelle phase, dite de stabilisation, précède la période de normalisation provoquée par la mortalité naturelle. Cette phase de stabilisation est liée au caractère tolérant du hêtre.

Il est vraisemblable que les développements en hauteur diffèrent d'une strate à l'autre avant que celles-ci ne forment des horizons physionomiquement distincts. En demi-lumière et à la lumière, les strates se sont distinguées, entre 5 et 15 ans, lorsque les distributions de hauteurs cumulées deviennent définitivement plurimodales (fig 8).

\section{Structure pluristrate et hiérarchie sociale}

Dans les peuplements adultes et les régénérations pluristrates, une position sociale est attribuée aux arbres de la strate supérieure, selon leur hauteur et leur forme. On distingue alors des individus dominants, dominés et surcimés (Delvaux, 1964 et 1975; Galoux, 1966; Lanier, 1986; Falcone et al, 1986; Zarnovican et Trencia, 1987; Schütz, 1990). Que représente alors la stratification et quelle est son influence sur la hiérarchie sociale ? Les dénominations de peuplement principal pour désigner la strate supérieure et de peuplement subordonné pour les autres strates (Falcone, 1985; Lanier, 1986; Schütz, 1990) semblent conférer à celles-ci une valeur de superstatuts sociaux.

Sans s'opposer à ce point de vue, nos observations apportent de nouvelles précisions. Contrairement à ce qui se passe dans les peuplements forestiers où les dominants sont souvent moins nombreux que les autres individus, au Lingas jusqu'à l'âge de 18 ans, on ne trouve pas une structure pyramidale des effectifs, tableau I. Si les strates dépendent les unes des autres pour la hauteur, chacune manifeste une certaine indépendance pour les effectifs. En sorte qu'un nombre comparable d'individus occupent l'espace qui leur est imparti dans chacune d'elles.

La variation discrète de la hauteur que l'on observe à la lumière (fig 1) a une signification importante car elle revèle qu'une norme est imposée à ce caractère dans chaque strate (Ford et Newbould, 1970). La relation asymptotique entre hauteur et diamètre au collet traduirait un niveau élevé d'organisation sociale à la lumière, alors que la relation quasi linéaire entre ces 2 caractères révèlerait un niveau d'organisation faible à nul en demi-lumière, où cependant il y a 2 strates, et à l'ombre. Nous retrouvons ici un raisonnement employé par des biologistes qui caractérisent une hiérarchie sociale par une relation asymptotique entre biomasse et effectifs, dite courbe de Lorentz (Weiner et Solbrig, 1984). Donc, une stratification ne constitue pas un argument suffisant pour assimiler les strates à des catégories sociales qui supposent une organisation contraignante dans laquelle la strate aurait une influence normative sur chacun de ses membres. 


\section{REMERCIEMENTS}

Travail réalisé avec l'aide de I'INRA, contrat "Amélioration, sylviculture et qualité du bois des feuillus précieuX», $n^{\circ} 1233 A$. Nous exprimons nos remerciements à Monsieur E Teissier-duCros et aux 2 lecteurs des Annales des Sciences Forestieres pour leurs remarques afin d'améliorer la rédaction de ce texte.

\section{RÉFÉRENCES}

Aussenac G, Ducrey M (1977) Étude bioclimatique d'une futaie feuillue (Fagus sylvatica $L$ et Quercus sessiliflora Salisb) de l'est de la France. I. Analyse des profils bioclimatiques et des caractères anatomiques et morphologiques de l'appareil foliaire. Ann Sci For 34, 265-284

Becker M (1981) Régénération naturelle. Conditions de germination des faînes, de survie et de croissance des semis, concurrence de la végétation. In : Le hêtre (Teissier-du-Cros $\mathrm{E}$, Le Tacon F, Nepveu G, Pardé J, Perrin R, Timbal J, eds) INRA, Département des Recherches Forestières, Paris, 224-228

Black JN, Wilkinson GN (1963) The role of time of emergence in determining the growth of individual plants in swards of subterraneum clover. Aust J Agric Res 14, 628-638

Bliss Cl, Reinker KA (1964) A lognormal approach to diameter distributions in even-aged stands. For Sci 10, 350-360

Comps B, Thiébaut B, Leroux A, Letouzey J (1988) Twenty year old beeches diversity in natural regenerations of Aigoual mountain (France): architecture, social regulation and genetic structures. In: Int Symp Silvic Improvment of Beech (Korpel S, Paule L, eds) IUFRO, June 1986; Zvolen, Czecoslovakia (à paraître)

Cuguen J, Merzeau D, Thiebaut B (1988) Genetic structure of the european beech stands (Fagus sylvatica L): F-statistics and importance of the mating system characteristics in their evolution. Heredity $60,91-100$

Delvaux J (1964) À propos de l'éclaircie des hêtraies en forêt de Soignes. Les aspects qualitatifs. Stn Rech Eaux For, GroenendaalHoeilaart, Trav Sér B, 30, $68 \mathrm{p}$
Delvaux J (1966) Contribution à l'étude de l'éducation des peuplements. II. A propos de distribution de fréquence de diamètres et de hauteurs. III. Essais préliminaires à l'étude du facteur compétition. IV. La compétition au niveau des classes sociales. Stn Rech Eaux For Groenendaal-Hoeilaart, Trav Sér B, 32, $46 p$

Delvaux J (1971) Contribution à l'étude de l'éducation des peuplements. $X$. Compétition interindividuelle chez le douglas. Stn Rech Eaux For Trav Sér B, 36, 23 p

Delvaux J (1975) Contribution à l'étude de l'éducation des peuplements. XIV. Acquisition du rang social dans les jeunes plantations d'épicéa. Stn Rech Eaux For, GroenendaalHoeilaart, Trav Sér B, 39, $32 \mathrm{p}$

Dupré S (1984) Morphologie et architecture des jeunes hêtres (Fagus sylvatica $\mathrm{L}$ ), influence du milieu et de la génétique. Mémoire $3^{\theta}$ année ENITEF, Nogent-sur-Vernisson, $102 p$

Dupré S, Thiébaut B, Teissier-du-Cros E (1986) Morphologie et architecture des jeunes hêtres (Fagus sylvatica $L$ ), influence du milieu, variabilité génétique. Ann Sci For 43, 85-102

Falconne $P$ (1985) Structure, croissance et aspects qualitatifs des plantations de Hêtre (Fagus sylvatica $\mathrm{L}$ ). Mémoire $3^{\theta}$ année, ENITEF, Stn sylvic prod INRA, CNRF (doc 85/ 01), Nancy, $90 \mathrm{p}$

Falcone $P$, Keller $R$, Le Tacon $F$, Oswald $H$ (1986) Facteurs influençant la forme des feuillus en plantation. Rev For Fr 38, 3, 315323

Ford ED (1975) Competition and stand structure in some even-aged plant monocultures. J Ecol 63, 311-333

Ford ED, Newbould PJ (1970) Stand structure and dry weight production throught the sweet chestnut (Castanea sativa Mill) Coppice Cycle. J Écol 58, 275-296

Galoux A (1966) La variabilité génécologique du Hêtre commun (Fagus sylvatica $L$ ) en Belgique. Stn Rech Eaux For, GroenendaalHoeilaart, Trav Sér A, 11, $121 \mathrm{p}$

Garnier M (1967) Climatologie de la France, sélection de données statistiques. Mémorial de la Météorologie Nationale, 50, $293 p$

Gauthier $C$ (1987) Amélioration des formes et comportements des plantations de Hêtre 
(Fagus sylvatica L) en Haute Normandie. Des solutions possibles. Mémoire $3^{\ominus}$ année, ENITEF, ENGREF, CIHEAM, FFSRC, $45 \mathrm{p}$

Hough AF (1932) Some diameter distribution in forest stands of northwestern Pennsylvania. $J$ For 30, 933-943

Hubert M (1968) Quels espacements doit-on adopter dans les plantations ? Bull Vul For $68,21 \mathrm{p}$

Hubert F (1984) Influence du froid, de la lumière et de stress hydrique sur la formation du bois de hêtre. Ann Sci For 41, 323-334

Johnson FL (1975) Size-class structure of three streamside forests. Am J Bot 62, 81-85

Koyama H, Kira T (1956) Intraspecific competition among higher plants. VIII. Frequency distribution of individual plant weight as affected by the interaction among plants. $J$ Inst Polytech Osaka Cy Univ 7, 73-94

Krahl-Urban $J$ (1962) BuchenNachkommenschaften. Allg Forst Jagdztg $133,29-38$

Lanier L (1981a) Conditions de germination des faînes, de survie et de croissance des semis. Coupes de mise en lumière. In : Le Hêtre (Teissier-du-Cros E, Le Tacon F, Nepveu G, Pardé J, Perrin R, Timbal J, eds) INRA, Dép Rech For, Paris, 239-241

Lanier L (1981b) Les dégagements et nettoiements en futaie feuillue. Rev For Fr $n^{\circ} s p 33$, $19-40$

Lanier L (1986) Précis de sylviculture. École Nat Génie Rural Eaux et Forêts, Nancy 468 p

Lee $Y$ (1971) Predicting mortality for even-aged stands of lodgepole pine. For Chron 47, 2932

Mohler CL, Marks PL, Sprugel DG (1978) Stand structure and allometry of trees during selfthinning of pure stands. $J$ Ecol $66,599-614$

Oswald H (1981) Régénération naturelle. Conditions de germination des faînes, de survie et de croissance des semis, densité de semis nécessaire à la réussite d'une régénération naturelle. In : Le Hêtre (Teissier-du-Cros E, Le Tacon F, Nepveu G, Pardé J, Perrin R, Timbal J, eds) INRA, Dép Rech For, Paris, 238-239

Polge H (1983) Essai de ligniculture du Hêtre à partir de régénération naturelles denses. Bull Tech ONF, 14, 11-17
Prodan M (1953) Die Verteilung des Vorrates gleichaltriger Holwaldbestände auf Durchmessersstufen. Allg Forst Jagdztg 93-106

Schnur GL (1934) Diameter distributions for oldfield loblolly pine stands in Maryland. $J$ Agric Res 49, 731-743

Schütz JP (1981) L'éclaircie sélective de Schädelin, évolution et pratique actuelle. Rev For Fr 33, $\mathrm{n}^{\circ} \mathrm{sp}, 7-18$

Schütz JP (1990) Sylviculture 1, principes d'éducation des forêts. Collection gérer l'environnement, Presses Polytechniques et Universitaires Romandes, Suisse, $243 p$

Suner A, Röhrig E (1980) Die Entwicklung der Buchennatur verjüngung in Abhängigkeit von der Auflichtung des Altbestandes. Forstarchiv 51, 145-149

Teissier-du-Cros E (1981) Principaux résultats en matière d'amélioration du hêtre. In:Le Hêtre (Teissier-du-Cros E, Le Tacon F, Nepveu $G$, Pardé J, Perrin R, Timbal J, eds) INRA, département des recherches forestières, Paris, 456-466

Teissier-du-Cros E, Thiébaut B (1988) Variability in beech: budding, height growth and tree form. Ann Sci For 45, 383-398

Thiébaut B (1979) Étude écologique de la hêtraie dans l'arc montagneux nordméditerranéen de la vallée du Rhône à celle de l'Ebre. Thèse Docteur d'État, Univ Sci Tech Languedoc, Montpellier, 267 p + annexes

Thiébaut B (1982) Observations sur le développement de plantules de hêtre (Fagus sylvatica L) cultivées en pépinière, orthotropie et plagiotropie. Can J Bot 60, 1292-1303

Thiébaut B (1985) Architecture des jeunes hêtres (Fagus sylvatica L). Bull Soc Bot Nord Fr 38, 7-25

Thiébaut B (1986) Approche des hêtres (Fagus sy/vatica L) : diversité intraspécifique, approche qualitative et quantitative. Nat Monspeliensia, Coll Int Arbre, 241-261

Thiébaut B, Comps B (1992) Influence de la sélection dans une régénération naturelle du Hêtre européen (Fagus sylvatica L). Bull Soc Étude Sci Nat Béziers (à paraître)

Thiébaut B, Payri C, Vigneron P, Puech S (1981) Observations sur la croissance et la floraison du hêtre (Fagus sylvatica L). Nat Monspelliensa Sér Bot, 48, 1-25 
Thiébaut B, Cuguen J, Dupré S (1985) Architecture des jeunes hêtres Fagus sylvatica $L$. Can J Bot 63, 2100-2110

Toda R (1963) Mass selection and heritability studies in forest tree breeding. World Consult For Genet Tree Improvement, Stockholm 2330 août, $7 p$

Urechiatu M (1986) Aspecte privind cultura fagulni in pepiniera (some aspects on Beech plantation in nurseries. Rev Padurilor 101, $71-76$

Van Miegroet M, Lust N (1972) Untersuchungen über die Entwicklungsdynamik von Eingebut- teten Aufforstungen von korsikanischen Schwarzföhre. Sylva Gandavensis, 31

Weiner RJ, Solbrig OT (1984) The meaning and measurement of size hierarchies in plant populations. Oecologia 61, 334-336

Yoda K, Kira T, Ogana H, Hozumi H (1963) Self thinning in overcrowded pure stands under cultivated and natural conditions. J Biol Osaka Cy Univ 14, 107-128

Zarnovican R, Trencia J (1987) Croissance de l'érable à sucre et du hêtre à grandes feuilles selon la position sociale dans le peuplement. Ann Sci For 44, 211-225 